\title{
A Bio-Inspired Distributed Clustering Algorithm for Wireless Sensor Networks
}

\author{
Charalambos Charalambous \\ charalambos@tamu.edu \\ Shuguang Cui \\ cui@tamu.edu \\ Department of Electrical \& Computer Engineering \\ Texas A\&M University \\ College Station, TX 77843
}

\begin{abstract}
Wireless sensor networks (WSNs) have emerged in strategic applications such as target detection, localization, and tracking in battlefields, where the large-scale nature renders centralized control prohibitive. In addition, the finite batteries in sensor nodes demand energy-aware network control. In this paper, we propose an energy-efficient topology management model that allows clustered nodes to act upon imminent targets in a purely distributed and autonomous fashion, which is inspired by the biological inter-cell lateral induction models. In particular, nodes in the target vicinity collaborate to form clusters based on their relative observation quality values. The energy efficiency of the proposed approach is examined against reference protocols.
\end{abstract}

\section{Categories and Subject Descriptors}

C.2.1 [Computer-communication Networks]: Network Architecture and Design—network topology, distributed networks

\section{General Terms}

Algorithms, Design

\section{Keywords}

Bio-inspired, sensor networks, distributed

\section{INTRODUCTION}

Recent progresses in wireless communications and electronics have facilitated the development of tiny multi-functional sensor nodes that are low-cost, low-power, and capable of communication in short distances [1]. These tiny sensor nodes consist of components responsible for sensing, data processing, and wireless communications. Their main tasks are to sense physical phenomena, process data, and forward useful information to a fusion center.

Permission to make digital or hard copies of all or part of this work for personal or classroom use is granted without fee provided that copies are not made or distributed for profit or commercial advantage and that copies bear this notice and the full citation on the first page. To copy otherwise, to republish, to post on servers or to redistribute to lists, requires prior specific permission and/or a fee.

WICON'08 November 17-19, 2008, Maui, Hawaii, USA.

Copyright 2008 ACM Copyright 2008 ICST 978-963-9799-36-3 ...\$5.00.
Sensor networks are composed of a large number of sensor nodes that are densely deployed over the area where targetsof-interest may appear. Their large-scale nature demands a high level of self-organization [18], where the participating entities configure themselves into a networking structure that requires minimum central management. In addition, they interact directly with neighboring nodes and constantly react to changing dynamics in their local surroundings. Such self-organizing systems typically feature flexibility, adaptiveness, robustness, and scalability [18]. The requirement of self-organization in WSNs favors distributed protocols that allow sensor nodes, or clusters of sensor nodes, to perform localized sensing and processing $[1,2]$. The absence of a central authority means that the sensor nodes enjoy the sovereignty to decide upon their own destiny, but should also bear the obligation to collaborate with other sensor nodes. In particular, by the cooperative effort the system can achieve better sensitivity and noise immunity via averaging across both space and time [2]. This is the key for success in large sensor networks since each individual node is limited in sensing range, transmit power, and processing capability $[6,22]$.

Many topology control approaches currently available are centralized with inhibitive complexity. This renders them inappropriate for large-scale networks that may operate under hostile conditions where connections to a central controller are rarely guaranteed. Even for networking protocols that are optimized for distributed implementations, there exist considerable drawbacks. The most notable one is the scalability issue: As the number of nodes increases, the performance deteriorates at a faster pace. The result is that even the most advanced available ad hoc protocols can only support dozens of nodes. This calls for the design of protocols that could handle wireless sensor networks of perhaps millions of nodes in an efficient manner. The fundamental reason for bad scalability is the lack of distributiveness. Many current algorithms, while being designed to be distributed, still possess some central elements in order to maintain a certain level of functionality for the protocol as a whole. The effect of not having a purely distributed protocol, illustrated by the issues of scalability, can be devastating on the practical design of a large-scale sensor network.

Among topology control approaches in sensor networks, clustering is an elegant way to address key issues such as coverage, connectivity, and energy conservation. As discussed above, distributed topology control is the only option for large-scale sensor networks. Most existing clustering algorithms are designed to achieve certain distributive- 
ness and can be classified into two main categories: datatransmission- oriented, which focus on inter- and intra-cluster routing to improve energy efficiency $[4,8,19,24]$; and applicationoriented, which aim at efficient target detection, tracking, and information aggregation $[9,12,21]$. There are also some algorithms that jointly consider both of these two aspects via heuristic approaches [20,26].

As we discussed over the issues of current topology control protocols, the existing clustering algorithms have scalability issues, especially when energy efficiency is the main design concern. It remains as a challenging problem how to autonomously form energy-efficient clusters around targets in a large-scale sensor network. Given the unsuccessful history in achieving the above goal, we have to challenge the way that traditional techniques tackle these issues. An attractive approach stems from biological research, where researchers found that living organisms consist of billions of networked cells interacting with each other in a remarkably harmonic way. Comparing the robust biological inter-cell networks with the struggling electronic networks, the contrast is clear: While current sensor network protocols suffer from scalability and efficiency issues, inter-cell biological networks exhibit purely distributed behavior, stability, high efficiency, and self-healing capabilities. In particular, although in the human body the main activities appear to be controlled by our brain in a centralized manner, it is interesting to note that the development process through which a body grows from several stem cells into a complex structure is solely controlled by distributed mechanisms. More surprisingly, even in the matured body, many activities such as wound healing are controlled by local cell clusters inside the corresponding tissues $[3,14]$.

Therefore, while the design of sensor networks is trying to overcome the issues that we mentioned, the biological intercell networks already possess all the elements that we seek. Specifically, these networks are purely distributed in nature, highly efficient, and enjoy autonomous reconfiguration. The following question then arises naturally: Could we design autonomous and distributed large-scale sensor networks by studying and learning from their biological inter-cell counterparts that have been polished by natural selection for millions of years? Thus inspired, we seek to design networking protocols via the methodology motivated by recent biological results, which indicate that billions of cells in organisms autonomously control their growth and interactions in both collaborative and competitive manners. Therefore, in this work we attempt to map inter-cell interactions in a living organism to inter-node dynamics in a sensor network, in the pursuit of efficient and distributed topology control protocols.

The rest of the paper is organized as follows. Section 2 provides some biology background that helps us understand the basis of the proposed model. Section 3 formally defines the networking problem that we address, and the corresponding mathematical formulations. In Section 4 we provide and discuss simulation results to verify our model, and in Section 5 we conclude our work and identify open challenges for future research.

\section{INTER-CELL BIOLOGICAL MODELS}

Before examining the biological context of the model that we propose, we first point out that topology control in sensor networks involves high-level strategic clustering and duty assignment for individual nodes. Specifically, multiple sensor nodes at a particular geographic location usually need to collaborate on a common task, such as target detection or tracking. Meanwhile, sensor nodes within the same cluster may compete for different assignments such as active sensing, information relaying, or just sleeping (in order to save resources, or to act as backups). The clustering process implies the collaborative relationship among sensor nodes, while the functional differentiation process demonstrates the competitive interaction among nodes. Such dynamics reverberates those of the biological development processes of some epithelium cells: Biologists found that during the chick inner ear development, Notch protein [7] first drives multiple cells in the same area to adopt similar characters to form a prosensory patch, and then mediates the hair cell versus supporting cell differentiation within the patch. Due to space limitations, in this paper we focus on the collaborative clustering aspect of network topology control, and we will examine the competitive feature in the subsequent work.

Inter-cell signaling is essential in the development of biological multi-cell systems, and generally involves the production of ligand - a small signaling molecule that binds to a protein or receptor - by the transmitting cells and its detection by specific receptors expressed by receiving cells, where the ligand is the mediator of the signal [13]. Juxtacrine signaling is a special class of inter-cell signaling, where ligands anchored in the membrane of a cell bind to and activate receptors on the surface of immediately neighboring cells [13]. As a result, signaling within a tissue can only occur between cells that are in direct contact with each other. With suitable feedback between receptor activation and expression levels of ligand, juxtacrine signaling is an efficient mechanism for the long-range propagation of localized signals, and thus the generation of spatiotemporal patterns [13].

A particularly well-documented juxtacrine signaling scheme is the Delta-Notch signaling $[5,7,13,16]$, where the activation of the Notch pathway (the receptor) by Delta (the ligand) affects the Delta activity of the receiving cell. Since the transmitting cell is also one of the neighboring cells of the receiving cell, the affected Delta activity in the receiving cell will be fed back to the receptors of the transmitting cell, leading to interactive closed-loop dynamics. As such, Delta-Notch signaling between cells is the main controlling scheme for cell pattern generation. Depending on the nature of the Delta-Notch feedback loops among neighboring cells, the pattern-generating processes can be classified into two different categories [7, 13, 17]: lateral induction and lateral inhibition.

Lateral induction, which is the main focus of this paper, is a process in which a cell heading for a particular fate induces its neighbors to adopt the same fate: If the Notch activation up-regulates Delta activity in the receiving cell, then this phenomenon propagates to neighboring cells, stopping only when this externally-driving up-regulation is beaten by internal degradation factors. Eventually a certain region is formed where all the cells in it achieve saturated Delta expressions, which corresponds to the formation of a functional patch of cells in the early stage of biological body development. Therefore, the main characteristic of the lateral induction mechanism is a feedback loop that is capable of amplifying initial similarities in the parameters of cells and their neighbors, such that homogeneous spatial patterns are generated. Figure 1 shows the feedback in lateral induction 


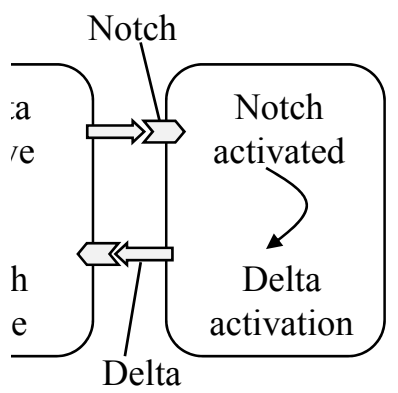

Figure 1: The feedback loop in lateral induction for a two-cell system: Reception of induction (Notch activation) increases the ability to deliver induction (Delta activity).

for a system of just two cells: Active Notch within a cell indicates reception of induction from the neighboring cell, which in turn up-regulates Delta activity in the former cell, increasing its ability to deliver induction to its neighbor, and so on.

Theoretical biologists and mathematicians have successfully modeled the Delta-Notch signaling process by sets of coupled ordinary differential equations (ODEs) $[5,13,15$, 23]. We consider the simple model in [5], where for the $i^{\text {th }}$ cell, $n_{i}$ denotes the levels of Notch activity, and $d_{i}$ denotes the levels of Delta activity. The following set of ODEs governs the behavior of the $i^{\text {th }}$ cell:

$$
\begin{aligned}
& \dot{n}_{i}=f\left(\bar{d}_{i}\right)-n_{i} \\
& \dot{d}_{i}=\mu\left\{g\left(n_{i}\right)-d_{i}\right\},
\end{aligned}
$$

where $\mu$ is a positive constant, $\bar{d}_{i}$ represents the average Delta activity at the neighbors of the $i^{\text {th }}$ cell, and $f\left(\bar{d}_{i}\right)$ is a function representing the production rate of the Notch activity in the cell, in response to the increasing amounts of Delta activity in neighboring cells. In particular, the function $f\left(\bar{d}_{i}\right)$ models the effect of either lateral induction or lateral inhibition jointly with the function $g\left(n_{i}\right)$. For example, in [5] the related functions are given by

$$
f(x)=\frac{x^{k}}{a+x^{k}}, \quad g(x)=\frac{1}{1+b x^{h}},
$$

where $a$ and $b$ are positive constants, $k \geq 1$, and $h \geq 1$.

\section{BIO-INSPIRED NETWORKING MODEL}

In this section, we propose a networking model in an attempt to mimic the behavior of biological inter-cell networks. In particular, sensor nodes collaborate to construct a functional cluster via lateral induction in a purely distributed fashion, as shown in Figure 2. In the context of target detection, the traditional cluster formation is usually based on the absolute local node observation quality values. However, due to the random noise in the observation field, some scattered nodes that are far away from the target may be included in the cluster, as long as they have good a observation quality value. As such, a non-compact cluster may be formed, which is not energy efficient in terms of data routing at later stages of networking operations. Bearing this in mind, our goal is to achieve compact clusters via the biological lateral induction model in a purely distributed manner.

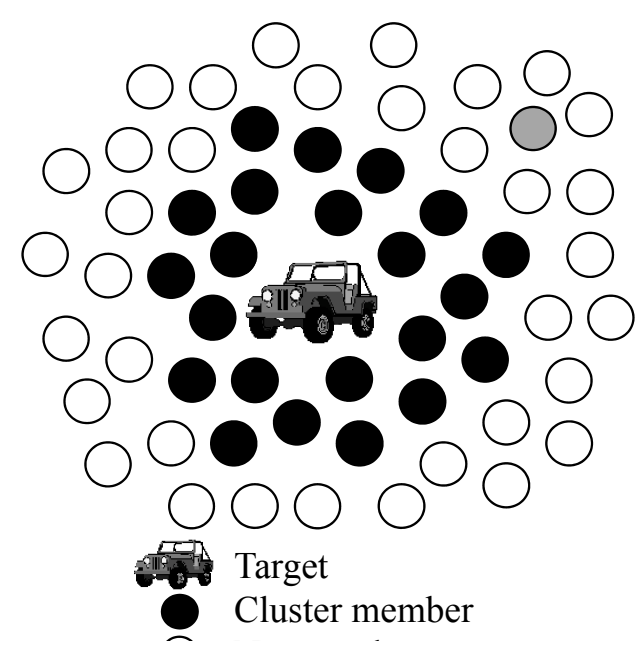

Figure 2: Laterally-induced cluster: Sensor nodes with relatively good observation quality values collaborate to form a cluster. Isolated nodes with good observation quality values are excluded.

\subsection{PROBLEM DEFINITION}

The specific application that we consider here is energyefficient target detection in a WSN, where a large number of energy-healthy nodes are randomly deployed into the field of interest. Immediately following their deployment, a preliminary hand-shaking mechanism is enforced such that sensor nodes could determine their neighborhood. Specifically, each node broadcasts a "hello" message and waits for replies from the nodes within its transmission range. Naturally, the number of replies that a node receives indicates the number of its neighbors. Since our model relies heavily on neighbor interactions, we assume that the initial topology constructed is a connected graph, i.e., there exists a communication route (which is usually multi-hop) connecting each node to any other node in the field. In idle status, all nodes are in "sleep" mode in order to conserve energy. During "sleep" mode, functions such as signal processing, decision-making, and neighbor communications are inactive. However, we assume that the basic sensing at each node is constantly being performed by the sensors. In the event that a target appears imminent, the nodes whose sensing signals surpass a certain threshold "wake up". At this moment, the clustering mechanism via lateral induction is invoked. The awaken nodes contact their neighbors and exchange the necessary information to run the induction model, and further act according to the evolution of the model parameters. Once the cluster is formed, the active nodes construct a minimum spanning tree (MST) to route their data to the clusterhead. For the time being, we assume that the clusterhead is the node with the highest observation quality of the target, which is selected by a particular mechanism (e.g., the competitive lateral inhibition algorithm that we skipped here). When the clusterhead gathers all the sensing reports from the active cluster members, it forwards, after some appropriate processing, the data to a fusion center that is responsible for final data fusion and analysis.

The design of inter- and intra-cluster communication pro- 
tocols, neighborhood discovery, and tree construction are beyond the scope of this paper, and we therefore assume that their mechanisms are predefined. To conform to the juxtacrine signaling models, we also assume that sensor nodes only exchange parameter values with their direct neighbors, thus ensuring that decisions on their fate are performed locally and distributively. Other assumptions we make are that the nodes are immobile and there is a single and stationary target in the network. In addition, the observation signal strength decays smoothly as the nodes are located further away from the target, but the observation quality values may be random due to the random strength of observation noises at different nodes.

\subsection{MATHEMATICAL FORMULATION}

We model the clustering process via lateral induction with a set of two differential equations for each sensor node, with a system analogous to the biological counterpart in [5]. For the $i^{\text {th }}$ node, let us denote with $q_{i}(t) \in[0,1]$ the time-evolving relative observation quality value of a node in reference to its neighbors, where $\left.q_{i}(t)\right|_{t=0}$ is the absolute observation quality value of the node, reflecting the signal-to-noise ratio (SNR) observed over the target. We assume that the SNR reading of each node has been normalized to be in the range [0, $1]$, according to a predefined maximum SNR reading. Let $s_{i}(t) \in[0,1]$ denote the time-evolving cluster membership indicator, where steady state values above a certain threshold mean that the node is selected as a cluster member and values below that threshold indicate that it is not selected. Specifically, the system of ODEs for node $i$ is given by

$$
\begin{aligned}
& \dot{q}_{i}=-q_{i}+f_{s}\left(s_{i}\right) \\
& \dot{s_{i}}=\mu\left\{-s_{i}+f_{\bar{q}}\left(\bar{q}_{i}\right)\right\},
\end{aligned}
$$

where $\mu$ is a positive constant, and the functions $f_{s}\left(s_{i}\right)$ and $f_{\bar{q}}\left(\bar{q}_{i}\right)$ are in the form of $f(x)$ defined in (1), with $a$ and $k$ particularly chosen for each function. The notation $\bar{q}_{i}$ indicates the average relative observation quality value over all the neighbors of the $i^{\text {th }}$ node. In particular, with $\mathcal{N}_{i}$ denoting the set of all the neighbors of node $i$, we have

$$
\bar{q}_{i}=\frac{1}{\left|\mathcal{N}_{i}\right|} \sum_{j} q_{j}, \forall j \in \mathcal{N}_{i},
$$

where $\left|\mathcal{N}_{i}\right|$ stands for the cardinality of the set $\mathcal{N}_{i}$.

The system of ODEs shown in (2) is a continuous-time model. Since in a sensor network protocols have to be executed in a discrete-time manner, we need to transform the above equations into difference equations. There are numerous methods to transform differential equations to difference equations. Here, we choose the Forward Euler method [10] for the convenience of analysis. According to this method, the derivative $y^{\prime}(t)=f(t, y(t))$ can be approximated as

$$
y^{\prime}(t)=\frac{y(t+T)-y(t)}{T},
$$

with $T$ being the step size. By rearranging parameters, the above equation gives:

$$
y(t+T)=y(t)+T f(t, y(t)) .
$$

We therefore construct appropriate sequences and index the time by $1,2, \ldots, n, n+1, \ldots$, at integer multiples of $T$, and we then solve for $y$ according to the iterative evolution of the equation

$$
y_{n+1}=y_{n}+T f\left(t_{n}, y_{n}\right)
$$

where $t_{n+1}=t_{n}+T$. We denote by $q_{i, n}$ the relative observation quality value of the $i^{\text {th }}$ sensor node at iteration step $n$, and by $s_{i, n}$ the cluster membership indicator of the $i^{\text {th }}$ node at iteration step $n$. Hence, the equations in (2) can be transformed into a set difference equations for each node:

$$
\begin{aligned}
& q_{i, n+1}=q_{i, n}+T\left\{f_{s}\left(s_{i, n}\right)-q_{i, n}\right\} \\
& s_{i, n+1}=s_{i, n}+T \mu\left\{f_{\bar{q}}\left(\bar{q}_{i, n}\right)-s_{i, n}\right\} .
\end{aligned}
$$

The reasoning behind the above induction model is simple. First, it is highly likely that a sensor node whose neighbors have good observation quality values over the target, has a comparable observation quality value itself. Meanwhile, with energy conservation as one of the main design objectives, it is desirable to have a compact cluster that is more energy-efficient in data routing. As such, we want an induction model where a sensor node is more likely to be selected as a cluster member if its neighbors are selected, and viceversa. In addition, large-scale networks usually operate in noisy environments and thus the distribution of observation quality values across a network is random in nature. As a result, a far-away sensor node might have a much better observation quality value than all its neighbors. With our model, it is likely that this sensor node would be denied cluster membership if its neighbors have a low average observation quality value. On the other hand, a sensor node might be selected as a cluster member even though it might have a mediocre observation quality value, if it is located in a neighborhood of nodes with high observation quality values. With the above discussion, we see that the collaborative nature of the lateral induction process leads to compact clusters. Such clusters do not burden intra-cluster communications, by denying cluster membership to good but isolated - nodes, while favoring compact neighborhoods of good nodes. Note that we will have a subsequent lateral inhibition model that will force the cluster members to compete with each other based on their energy levels until a minimal subset of these nodes remains active (to satisfy a certain application requirement). The inactive cluster members will later have the chance to participate in the "rotation" mechanism to periodically become active. Such a next-stage topology optimization via lateral inhibition will be addressed in our future work.

Mathematically, the collaborative interaction among sensor nodes that is responsible for the cluster construction is mediated by the average relative observation quality value within the neighborhood of a particular node. This average value of the neighbors affects the cluster membership indicator value of that node. In turn, the node affects the cluster membership indicator values of its neighbors, and the feedback loop continues. For the model in (3), by adjusting the system constants $a$ and $k$ in the functions $f_{s}\left(s_{i}\right)$ and $f_{\bar{q}}\left(\bar{q}_{i}\right)$ accordingly, we can change the shape of these functions responsible for the evolution of the induction parameters. In this way, we can control the final cluster size in the steady state in order to fit the needs of a specific sensing application. For a more complex system model, some constants can be set to be dependent on other parameters and adapt to different dynamics across the network. For example, we could further alter the cluster size by having model parameters respond to the energy levels of the neighbors, which will be investigated in our future work in order to optimize the overall network energy efficiency.

Furthermore, the constant $\mu$ represents the ratio between 


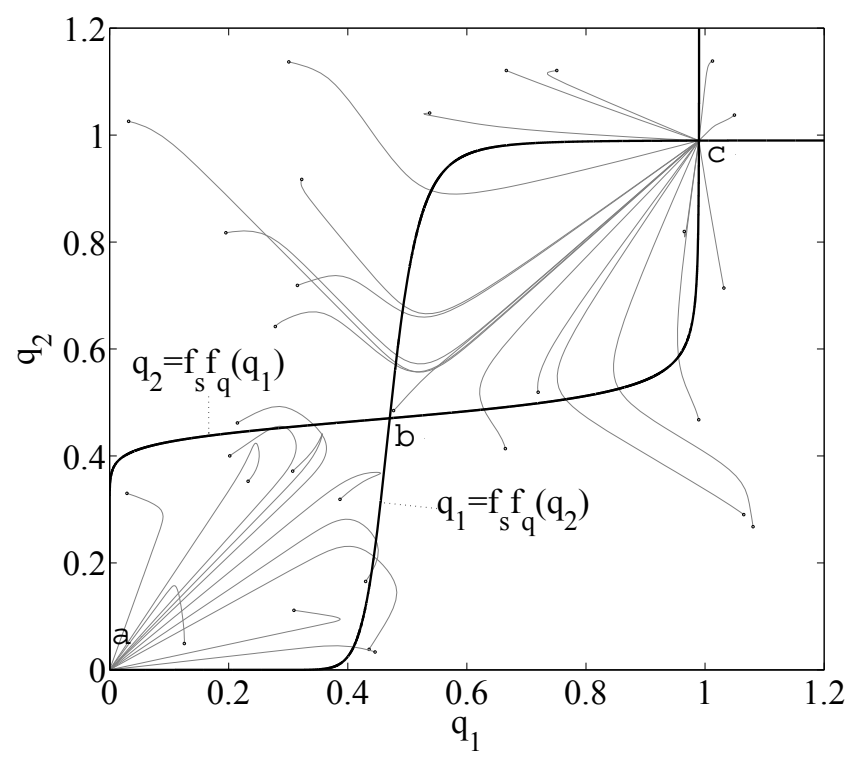

Figure 3: Phase plane for the simple system consisting of two nodes. There are three equilibrium points $\{a, b, c\}$, of which point $b$ is an unstable saddle point, and points a and $c$ are stable equilibrium points. For large values of $\mu$, it is the initial values of $q_{1}$ and $q_{2}$ that determine which of the two stable equilibrium points that the system settles in. Here $\mu=10$.

the decay rates of $q$ and $s$, and is therefore a measure of the relative time-scales over which the levels of the relative observation quality and the cluster membership indicator values vary [5]. Increasing the values of $\mu$ and/or $k$ increases the convergence speed [5]; however, extremely large values may lead to numerical issues in discrete implementations, which result in instability. Actually, the continuous-time biological induction model is inherently stable. Therefore, the increase in the values of the aforementioned parameters reduces the time taken for the final cluster pattern to emerge, while the upper limit for those parameters is set by some other biological mechanisms. In a wireless network though, operations are performed in discrete-time steps by nodes that have finite-bit accuracy. Therefore, selecting extremely large values for $\mu$ and/or $k$ might lead to instability caused by numerical issues. Theoretically, the particular choice of $\mu$ does not affect the possible steady states of the system, which are determined by the choices of $a$ and $k$ in the functions $f_{s}\left(s_{i}\right)$ and $f_{\bar{q}}\left(\bar{q}_{i}\right)$. However, when the system admits several equilibrium points, which of the steady states the system settles in is influenced by $\mu$ and other initial conditions.

For example, in a simple continuous-time system consisting of just two nodes, the equations in (2) become

$$
\begin{aligned}
& \dot{q_{1}}=-q_{1}+f_{s}\left(s_{1}\right), \dot{s_{1}}=\mu\left\{-s_{1}+f_{\bar{q}}\left(\bar{q}_{2}\right)\right\} ; \\
& \dot{q_{2}}=-q_{2}+f_{s}\left(s_{2}\right), \dot{s_{2}}=\mu\left\{-s_{2}+f_{\bar{q}}\left(\bar{q}_{2}\right)\right\},
\end{aligned}
$$

where the subscripts correspond to sensor nodes 1 and 2 . Equilibrium points of the system in (4) are given by

$$
\left(q_{1}^{*}, s_{1}^{*}, q_{2}^{*}, s_{2}^{*}\right)=\left(f_{s}\left(s_{1}^{*}\right), s_{1}^{*}, f_{s}\left(s_{2}^{*}\right), s_{2}^{*}\right),
$$

where $s_{1}^{*}$ and $s_{2}^{*}$ are fixed points of the composite function

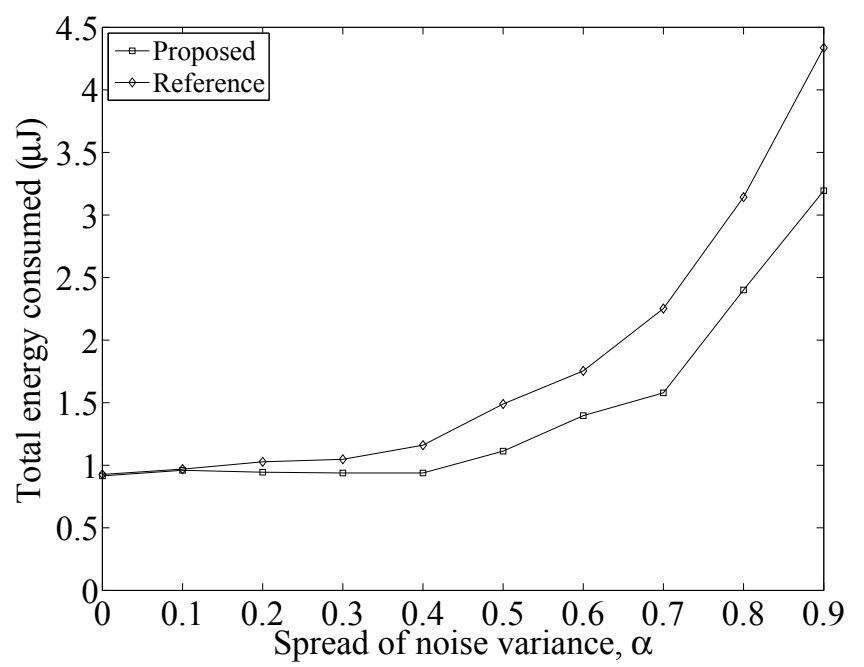

Figure 4: Total energy required for all the cluster members to forward their sensing reports to the clusterhead, over the spread of observation noise variance. A compact cluster requires less total energy than one which includes distant nodes.

$f_{\bar{q}} \circ f_{s} \circ f_{\bar{q}} \circ f_{s}$ with $s_{1}^{*}=f_{\bar{q}}\left(f_{s}\left(s_{2}^{*}\right)\right)$ and $s_{2}^{*}=f_{\bar{q}}\left(f_{s}\left(s_{1}^{*}\right)\right)$. For functions $f_{s}$ and $f_{\bar{q}}$ having the form of $f(x)$ in (1), there are three equilibrium points with $q_{1}^{*}=q_{2}^{*}$ and $s_{1}^{*}=s_{2}^{*}$. A steady state $x$ is linearly stable if $\left(f_{s} \circ f_{\bar{q}} \circ f_{s} \circ f_{\bar{q}}\right)^{\prime}(x)<1$, but unstable otherwise. Figure 3 shows the phase plane of the relative observation quality values for this two-node example. The dark-weighted lines indicate the nullclines $q_{1}=\left(f_{s} \circ f_{\bar{q}}\right)\left(q_{2}\right)$ and $q_{2}=\left(f_{s} \circ f_{\bar{q}}\right)\left(q_{1}\right)$, on which $\dot{q}_{1}=0$ and $\dot{q}_{2}=0$ respectively. The various trajectories in the phase plane of Figure 3, plotted for different initial conditions of $q_{1}$ and $q_{2}$, show that there is one unstable and two stable equilibrium points for this two-node system. The three equilibrium points are $\mathrm{a}=(0,0), \mathrm{b}=(0.4705,0.4705)$, and $\mathrm{c}=(1,1)$. In particular, the equilibrium points a and $c$ are stable, while point $\mathrm{b}$ is an unstable saddle point. As discussed before, the location of the equilibrium points depends on the parameters $a$ and $k$. In this two-node example, $a=0.01$ and $k=3.5$ for the function $f_{s}, a=0.01$ and $k=7.5$ for the function $f_{\bar{q}}$, and $\mu=10$.

We next discuss how to achieve one of the two stable equilibrium points in this example. When $\mu \gg 1$, we can make the quasi-steady-state assumption [5] that

$$
f_{\bar{q}}\left(q_{2}\right)-s_{1}=0, \quad f_{\bar{q}}\left(q_{1}\right)-s_{2}=0,
$$

and thus the system in (4) reduces to

$$
\dot{q}_{1}=-q_{1}+\left(f_{s} \circ f_{\bar{q}}\right)\left(q_{2}\right), \quad \dot{q}_{2}=-q_{2}+\left(f_{s} \circ f_{\bar{q}}\right)\left(q_{1}\right) .
$$

The phase plane in Figure 3 shows that when $\mu \gg 1$ it is the initial values of $q_{1}$ and $q_{2}$ that determine which of the two stable homogeneous equilibrium points will be attained eventually. Analogous analysis for $\mu \ll 1$ can be performed, leading to the conclusion that it is the initial values of $s_{1}$ and $s_{2}$ that determine the final steady state (results not shown). Hence, we have chosen a value for $\mu=10 \gg 1$ for the simulations, reflecting our desire to decide upon cluster membership based on the relative observation quality values, i.e., the $q_{i}$ 's. 


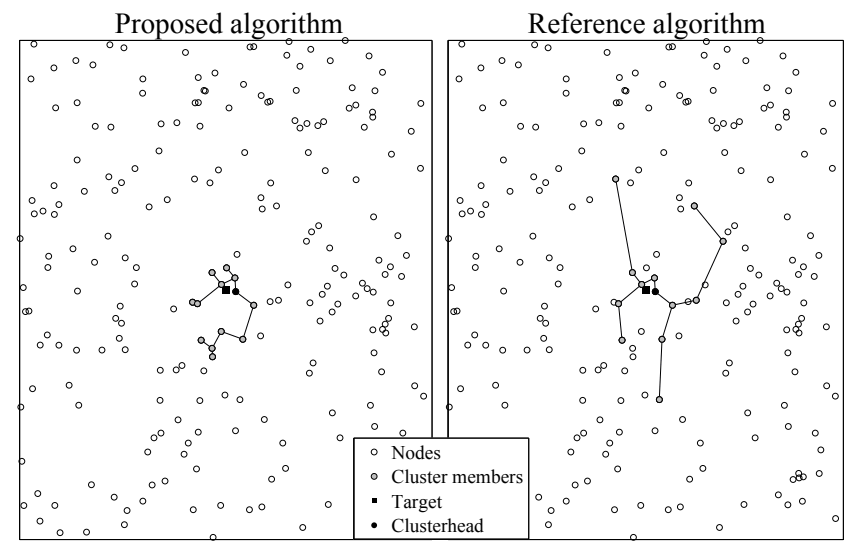

Figure 5: The proposed algorithm produces a more compact cluster than the reference algorithm. There are 13 cluster members in both cases. Lines connecting nodes stand for the MST routes.

\section{SIMULATION RESULTS}

The performance metric by which we assess our algorithm is the total energy consumed by the cluster as a whole to deliver sensing reports to the clusterhead, i.e., the total energy required for each cluster member to forward its sensing report to the clusterhead via a route constructed in the MST. We assume that the sensor node with the best observation quality value is chosen to serve as the clusterhead and is therefore the root of the MST constructed by the Kruskal algorithm [11]. The weight function for the Kruskal algorithm is represented by the square of the distance between the cluster members. The initial absolute observation quality value of the $i^{\text {th }}$ node depends on the distance from the target $r, d_{i, r}$ :

$$
q_{i}(0)=\frac{e^{\frac{-d_{i, r}^{2}}{2 \sigma^{2}}}}{z_{i}},
$$

where $\sigma$ is a deviation measure of spread of the observation quality distribution. The parameter $z_{i}$ denotes the variance of the observation noise and is assumed to be uniformly distributed with mean 1 between $[1-\alpha, 1+\alpha]$, where $\alpha$ stands for the spread of the noise variance. Essentially, $q_{i}(0)$ represents the normalized SNR at each sensor node and $\left.q_{i}(t)\right|_{t>0}$ represents the relative normalized SNR. We assume that $s_{i}(0)=0, \forall i$ (even though the initial value of the cluster membership indicator of each node does not affect the final steady state when $\mu \gg 1$, as discussed in Section 3.2). The energy required to transmit $b$ bits of data from one node directly (i.e., with just a single hop) to another that is located at a distance $d$ away, is defined as:

$$
E_{T X}(k, d)=\epsilon b d^{2},
$$

where $\epsilon$ is the transmission system constant, according to the first-order radio model in [8].

We compare our algorithm to a reference scheme loosely based on the initial stage of the Dynamic Convoy Tree-based Collaboration (DCTC) algorithm [25], which mainly concentrates on a moving target and creates a tree of nodes surrounding it as the target traverses across the network. The root of the tree is responsible for reconfiguring the tree, by adding or pruning nodes as the target moves. The initial

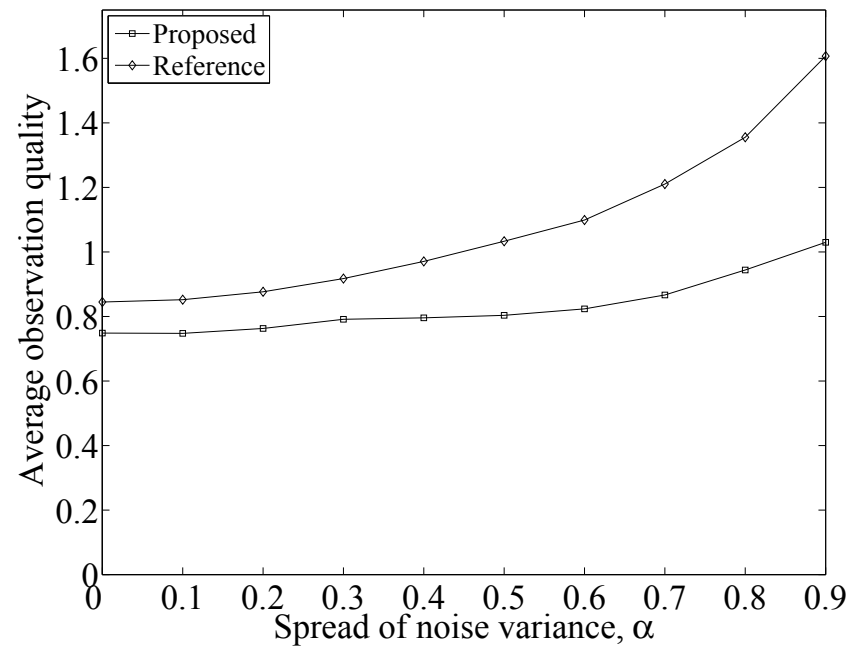

Figure 6: Average observation quality of all the cluster members as a function of the spread of observation noise variance. Energy efficiency within a compact cluster comes at a certain expense of observation quality.

tree construction of the DCTC algorithm is achieved by the nodes that are awake and close to the target.

We simulated in MATLAB for different levels of noise variance spread for a randomly-deployed network over a square field of side length $10 \mathrm{~m}$. There are 220 nodes in the field, and each node has a transmission range of $1.5 \mathrm{~m}$. In addition, the target is situated at the center of the field, $\sigma=2$, each node has a 320-bit sensing report to forward to the clusterhead, and $\epsilon=100 \mathrm{pJ} / \mathrm{bit} / \mathrm{m}^{2}$ [8]. We simulated 200 random network configurations for each of 10 different levels of $\alpha$. We set the threshold for cluster membership to be an indicator value greater or equal to 0.9 . In this paper, we adopt parallel synchronous updates of the state vectors across the networking nodes. We will investigate the asynchronous case in the future research.

In both of the schemes, once the cluster members are selected, we assume that all nodes increase their transmission power until they are neighbors with all other cluster members. Then, the MST is formed by the Kruskal algorithm. Once the MST is constructed, the nodes readjust their transmission powers in order to conform to the MST routes. In other words, they reduce their transmission power by the necessary proportion so as to communicate directly with the node that is identified as the next hop in the corresponding MST route. Energy is expended according to the model in (5).

Under low-noise-spread conditions the two algorithms perform comparably, as shown in Figure 4. As the spread of the observation noise variance increases, leading to nodes being increasingly misled about their actual distance from the target, the difference in performance becomes clear. Figure 4 shows that the proposed algorithm expends less overall energy to forward messages from the sensor nodes to the clusterhead for the same number of cluster members. This implies that, via inter-node collaboration, the induction clustering technique constructs a more compact cluster, as seen in Figure 5. But this comes at a certain expense of obser- 


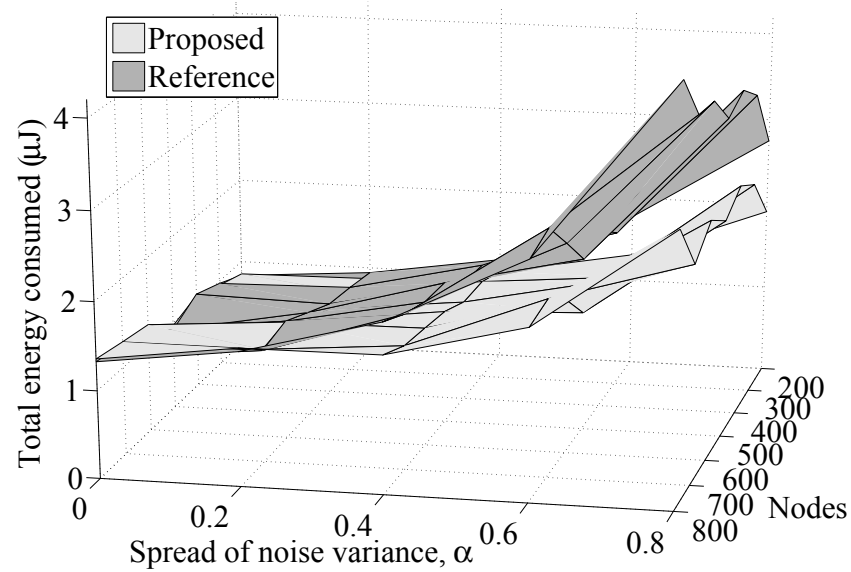

Figure 7: Total energy required for all the cluster members to forward their sensing reports to the clusterhead, as a function of the number of nodes in the network and the spread of observation noise variance.

vation quality. As Figure 6 shows, for the same number of cluster members, the average observation quality values of the cluster members are lower than those of the reference scheme. This is due to the fact that the lateral induction process might force sensor nodes within a compact neighborhood into joining the cluster as a result of "peer pressure", even though they may not have comparably high observation quality values. At the same time, the induction process might exclude sensor nodes with very good observation quality values from joining the cluster, if they are isolated in a vicinity of nodes with poor observation quality values. By adjusting our model constants, we could control the tradeoff between the gained energy efficiency and the lost average observation quality. Furthermore, the results depicted in Figure 7 show that as the network size and the spread of observation noise variance increase, the proposed algorithm improves the relative energy efficiency.

In order to examine the effect of the constant $\mu$ on convergence speed, we simulated for a network consisting of 200 nodes, deployed in a square field of side length $10 \mathrm{~m}$. Each node has a $1.5 \mathrm{~m}$ transmission range. The step size in (3) is $T=0.05$. We averaged the number of steps necessary for each node to settle within $5 \%$ and $1 \%$ of its final values, over 200 different random network configurations, for 11 values of $\mu$ at each configuration. Simulations were performed for values of $\mu \geq 10$ to conform to the discussion in Section 3.2. Results in Figure 8 show that the average number of steps needed to reach the steady state decreases as $\mu$ increases, even though the incremental speed improvement decreases over higher values of $\mu$.

\section{CONCLUSION}

In this paper, we propose a distributed clustering algorithm for WSNs based on the biological lateral induction model. Simulations show that, by fostering collaborative interaction through lateral induction, our clustering model increases the energy efficiency through the construction of compact clusters. In addition, this procedure is purely distributed; all decisions by the nodes are performed locally by

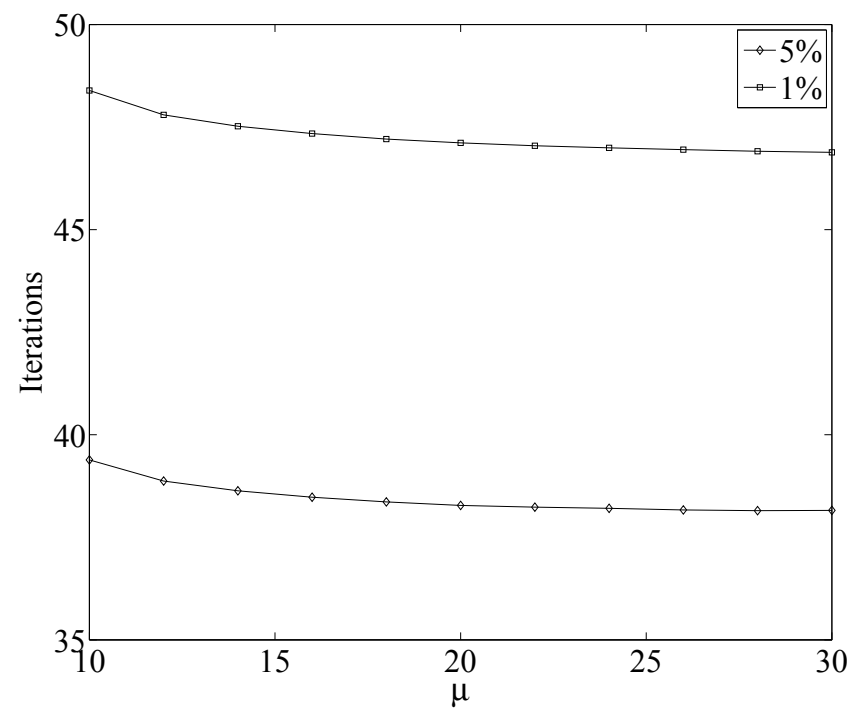

Figure 8: Average number of iteration steps needed to settle within $5 \%$ and $1 \%$ of the steady state, versus the value of the constant $\mu$. The system settles faster as $\mu$ increases.

observing their neighboring environment.

While the lateral-induction-based clustering algorithm operates in an energy efficient fashion, some important issues still need to be addressed in order to further improve its performance. For example, we need to explicitly define a clusterhead election procedure. Additionally, the clustering algorithm can be further optimized by carefully selecting model constants, or by employing more complex functions. Furthermore, combining the lateral induction clustering process with a subsequent lateral inhibition phase that will select a subset of nodes within the cluster is part of our immediate research goals. The lateral-inhibition-based competition will encourage a rotation mechanism within the cluster for further improving the energy efficiency.

\section{ACKNOWLEDGMENTS}

This work is partially supported by NSF under Grant No. 0726740 and DoD under Grant HDTRA-07-1-0037.

\section{REFERENCES}

[1] I. F. Akyildiz, W. Su, Y. Sankarasubramaniam, and E. Cayirci. Wireless sensor networks: A survey. Computer Networks, 38(4):393-422, March 2002.

[2] A. Arora, P. Dutta, S. Bapat, V. Kulathumani, H. Zhang, V. Naik, V. Mittal, H. Gao, M. Demirbas, M. Gouda, Y. Choi, T. Herman, S. Kulkarni, U. Arumugam, M. Nesterenko, A. Vora, and M. Miyashita. A line in the sand: A wireless sensor network for target detection, classification, and tracking. Computer Networks, 46(5):605-634, December 2004.

[3] A. Bailón-Plaza and M. C. H. van der Meulen. A mathematical framework to study the effects of growth factor influences on fracture healing. Journal of Theoretical Biology, 212(2):191-209, September 2001. 
[4] M. Chatterjee, S. K. Das, and D. Turgut. WCA: A Weighted Clustering Algorithm for mobile ad hoc networks. Cluster Computing, 5(2):193-204, April 2002.

[5] J. R. Collier, N. A. Monk, P. K. Maini, and J. H. Lewis. Pattern formation by lateral inhibition with feedback: A mathematical model of delta-notch intercellular signalling. Journal of Theoretical Biology, 183(4):429-446, December 1996.

[6] S. Cui, A. J. Goldsmith, and A. Bahai. Energy efficiency of MIMO and cooperative MIMO in sensor networks. IEEE Journal on Selected Areas in Communications, 22(6):1089-1098, August 2004.

[7] N. Daudet and J. Lewis. Two contrasting roles for notch activity in chick inner ear development: Specification of prosensory patches and lateral inhibition of hair-cell differentiation. Development, 132(3):541-551, February 2005.

[8] W. R. Heinzelman, A. Chandrakasan, and H. Balakrishnan. Energy-efficient communication protocol for wireless microsensor networks. In Proceedings of the 33rd Hawaii International Conference on System Sciences, pages 3005-3014, January 2000.

[9] G-Y. Jin, X-Y. Lu, and M-S. Park. Dynamic clustering for object tracking in wireless sensor networks. Lecture Notes in Computer Science, 4239:200-209, 2006.

[10] E. Kreyszig. Advance engineering mathematics. John Wiley \& Sons, Inc., New York, 1999.

[11] J. B. Kruskal. On the shortest spanning subtree of a graph and the traveling salesman problem. Proceedings of the American Mathematical Society, 7(1):48-50, February 1956.

[12] C-M. Liu, C-H. Lee, and L-C. Wang. Distributed clustering algorithms for data-gathering in wireless mobile sensor networks. Journal of Parallel and Distributed Computing, 67(11):1187-1200, November 2007.

[13] N. A. Monk, J. A. Sherratt, and M. R. Owen. Spatiotemporal patterning in models of juxtacrine intercellular signalling with feedback. Institute for Mathematics and Its Applications, 121:165-193, 2001.

[14] D. L. Nikolić, A. N. Boettiger, D. Bar-Sagi, J. D. Carbeck, and S. Y. Shvartsman. Role of boundary conditions in an experimental model of epithelial wound healing. American Journal of Physiology-Cell Physiology, 291(1):68-75, July 2006.
[15] M. R. Owen and J. A. Sherratt. Mathematical modeling of juxtacrine cell signaling. Mathematical Biosciences, 153(2):125-150, November 1998.

[16] M. R. Owen, J. A. Sherratt, and H. J. Wearing. Lateral induction by juxtacrine signaling is a new mechanism for pattern formation. Developmental Biology, 217(1):54-61, January 2000.

[17] E. Plahte. Pattern formation in discrete cell lattices. Journal of Mathematical Biology, 43(5):411-445, November 2001.

[18] C. Prehofer and C. Bettstetter. Self-organization in communication networks: Principles and design paradigms. IEEE Communications Magazine, 43(7):78-85, July 2005.

[19] M. Qin and R. Zimmermann. VCA: An energy-efficient Voting-based Clustering Algorithm for sensor networks. Journal of Universal Computer Science, 13(1):87-109, 2007.

[20] C. Sharp, S. Schaffert, A. Woo, N. Sastry, and C. Karlof. Design and implementation of a sensor network system for vehicle tracking and autonomous interception. In Proceedings of Second European Workshop on Wireless Sensor Networks, pages 93-107, January 2005.

[21] X. Wang, J-J. Ma, S. Wang, and D-W. Bi. Cluster-based dynamic energy management for collaborative target tracking in wireless sensor networks. Sensors, 7(7):1193-1215, July 2007.

[22] X. Wang and S. Wang. Collaborative signal processing for target tracking in distributed wireless sensor networks. Journal of Parallel and Distributed Computing, 67(5):501-515, May 2007.

[23] S. D. Webb and M. R. Owen. Oscillations and patterns in spatially discrete models for developmental intercellular signalling. Journal of Mathematical Biology, 48(4):444-476, April 2004.

[24] O. Younis and S. Fahmy. HEED: A Hybrid, Energy-Efficient, Distributed clustering approach for ad hoc sensor networks. IEEE Transactions on Mobile Computing, 3(4):366-379, October 2004.

[25] W. Zhang and G. Cao. DCTC: Dynamic Convoy Tree-based Collaboration for target tracking in sensor networks. IEEE Transactions on Wireless Communications, 3(5):1689-1701, September 2004.

[26] F. Zhao, J. Shin, and J. Reich. Information-driven dynamic sensor collaboration. IEEE Signal Processing Magazine, 19(2):61-72, March 2002. 